\title{
Advances in nonlinear dynamics of complex networks: adaptivity, stochasticity, and delays
}

\author{
Vladimir Nekorkin ${ }^{\mathrm{a}}$ and Vladimir Klinshov \\ Institute of Applied Physics of the Russian Academy of Sciences, Uljanova str. 46, \\ 603950 Nizhny, Novgorod, Russia
}

Received 24 October 2018

Published online 12 December 2018

Networks of interacting units are omnipresent in nature, technology, society, and economy. In recent decades, a tremendous progress in network science is observed due to the joint effect of data accumulation, development of computers, and emergence of novel concepts. The mankind gains understanding of more and more complex networks, the human genome and the connectome being the most striking examples. However, the complexity of networks may be related not only to their structure. The collective temporal behavior that the network demonstrates may be highly nontrivial even if its connectivity is relatively simple. This complex dynamics may arise due to the different factors related to the individual nodes as well as their interactions. The possible aspects of complexity may include adaptive connections, transmission delays, chaotic or stochastic dynamics of the nodes, etc.

The present EPJ special topic issue is devoted to the recent progress in dynamics of complex networks. The contributions collected here provide a broad picture of how various aspects of complexity may influence the collective behavior of networks. One of these aspects is variable or adaptive network connectivity. Connectivity evolving due to the network maturation or chemical action was shown to have dramatic effect on the dynamics of neural networks in vitro [1]. A model of the neural connectivity shaping due to the axonal growth was suggested, along with the theory of the network bursting behavior [2]. The influence of the plasticity type on the collective dynamics of neural networks was studied [3]. A concept of multilayer networks with adaptive connections was suggested as a novel framework for neural computations [4]. Adaptive connections were shown to provide a novel mechanism for the emergence of chimera states and hierarchical synchronized states [5].

If the nodes of a network are subject to stochastic input, this may cause qualitative changes in the collective behavior. The influence of noise may lead to switching between various levels of localized activity in neural networks [6]. In the combination with plasticity, the noise may induce two different types of switching: between the states with various activity and between the states with various spiking patterns [7]. Two different regimes of sequential escape behavior in coupled noisy systems were reported depending on the coupling strength [8]. The impact of noise on the dynamics of a hierarchical heteroclinic network was studied in reference [9].

Yet another aspect of complexity attracting attention of many researchers is delayed connections. Coupling delays may effectively control synchronization in networks of neural-like oscillators [10]. In combination with fluctuating topology, the

a e-mail: vnekorkin@neuron.appl.sci-nnov.ru 
effect of coupling delays on synchronization depends on the relation between the timescales in a nontrivial manner [11]. Synchronization may be also suppressed by the application on the delayed feedback, as shown for the human brain simulations [12].

Nontrivial collective behavior of different types is often seen in networks with various aspects of complexity. The influence of multiplex connectivity and coupling delays on the synchronization of chimeras was considered [13]. The emergence of solitary states in a network of chaotic units with nonlocal coupling was studied [14]. Collective irregular dynamics of balanced neural networks with delayed coupling was reported [15]. The intermittency in delay-coupled fast-slow systems generating extreme events was investigated [16]. The correlation between the nodes parameters and the connectivity was shown to cause hierarchical transitions in oscillatory networks [17].

A number of contributions are devoted to novel original methods applicable for the analysis of complex networks. A novel concept of the so-called mean complexity was applied for the analysis of network dynamics and revealing its topology features [18]. A method to recover the network connectivity from the observed dynamics was suggested [19]. New tools for the analysis of the synchronous states in networks with nonsmoothness and discontinuity were presented [20]. The conditions for the asymptotic periodicity in networks of degrade-and-fire oscillators were obtained [21]. An analytical method for delays reduction in networks was suggested [22].

We would like to thank all the authors for their contributions as well as the referees for their great job in reviewing the articles. We also thank all the members of EPJ ST, especially Sabine Lehr and Nicolas Puyaubreau, for hosting this issue and the support. Our special thanks go to Prof. Jürgen Kurths, whose help in the preparation of this issue cannot be overstated. We hope that the present issue will be helpful for the researchers interested in dynamics of complex networks and their applications.

\section{References}

1. E. Tibau, J. Soriano, Eur. Phys. J. Special Topics 227, 999 (2018)

2. P. Monceau, S. Métens, T. Fardet, R. Renault, S. Bottani, Eur. Phys. J. Special Topics 227, 1015 (2018)

3. A. Hernández, J.M. Amigó, Eur. Phys. J. Special Topics 227, 1029 (2018)

4. A.V. Andreev, E.N. Pitsik, V.V. Makarov, A.N. Pisarchik, A.E. Hramov, Eur. Phys. J. Special Topics 227, 1039 (2018)

5. D.V. Kasatkin, V.I. Nekorkin, Eur. Phys. J. Special Topics 227, 1051 (2018)

6. I. Franovic, V. Klinshov, Eur. Phys. J. Special Topics 227, 1063 (2018)

7. I. Bacic, S. Yanchuk, M. Wolfrum, I. Franovic, Eur. Phys. J. Special Topics 227, 1077 (2018)

8. P. Ashwin, J. Creaser, K. Tsaneva-Atanasova, Eur. Phys. J. Special Topics 227, 1091 (2018)

9. M. Voit, H. Meyer-Ortmanns, Eur. Phys. J. Special Topics 227, 1101 (2018)

10. V. Klinshov, L. Lücken, S. Yanchuk, Eur. Phys. J. Special Topics 227, 1117 (2018)

11. O. D'Huys, J. Rodríguez-Laguna, M. Jiménez, E. Korutcheva, W. Kinzel, Eur. Phys. J. Special Topics 227, 1129 (2018)

12. M. Mugnaine, A.S. Reis, F.S. Borges, R.R. Borges, F.A.S. Ferrari, K.C. Iarosz, I.L. Caldas, E.L. Lameu, R.L. Viana, J.D. Szezech, Jr. J. Kurths, A.M. Batista, Eur. Phys. J. Special Topics 227, 1151 (2018)

13. J. Sawicki, I. Omelchenko, A. Zakharova, E. Schöll, Eur. Phys. J. Special Topics 227, $1161(2018)$

14. N. Semenova, T. Vadivasova, V. Anishchenko, Eur. Phys. J. Special Topics 227, 1173 (2018) 
15. A. Politi, E. Ullner, A. Torcini, Eur. Phys. J. Special Topics 227, 1185 (2018)

16. A. Saha, U. Feudel, Eur. Phys. J. Special Topics 227, 1205 (2018)

17. O.V. Maslennikov, V.I. Nekorkin, Eur. Phys. J. Special Topics 227, 1221 (2018)

18. V. Afraimovich, A. Dmitrichev, D. Shchapin, V. Nekorkin, Eur. Phys. J. Special Topics 227, 1231 (2018)

19. N. Rubido, C. Masoller, Eur. Phys. J. Special Topics 227, 1243 (2018)

20. Y.M. Lai, R. Thul, S. Coombes, Eur. Phys. J. Special Topics 227, 1251 (2018)

21. B. Fernandez, Eur. Phys. J. Special Topics 227, 1267 (2018)

22. A. Wagemakers, J. Used, M.A.F. Sanjuán, Eur. Phys. J. Special Topics 227, 1281 (2018) 